\title{
Satisfacción Personal como Componente de la Calidad de Vida de los Adultos de Medellín
}

\author{
Personal satisfaction as a component of adult's quality \\ of life in Medellin
}

Doris Cardona y Héctor B. Agudelo

Facultad Nacional de Salud Pública, Universidad de Antioquia. doriscar@guajiros.udea.edu.co, bagudelo@guajiros.udea.edu.co

Recibido 7 Febrero 2007/Enviado para Modificación 7 Octubre 2007/Aceptado 21 Octubre 2007

\section{RESUMEN}

Objetivo Determinar la satisfacción personal como el principal componente de la calidad de vida de los hombres y las mujeres de 20 a 64 años de la ciudad de Medellín en el año 2005.

Métodos Estudio poblacional descriptivo transversal, con fuente de información primaria constituida por dos muestras: 659 hombres y 683 mujeres, que representaron a 523705 y 651704 respectivamente, según factores de expansión poblacional; seleccionados con muestreo complejo probabilístico, estratificado por estrato socioeconómico, por conglomerados y polietápico: manzanas, viviendas y personas.

Resultados La calidad de vida actual de la población adulta de la ciudad de Medellín está dada principalmente por los aspectos personales, económicos, entorno físico de la vivienda y seguridad social. Los aspectos analizados fueron valorados por encima de $75 \%$, los que mayor satisfacción generan son las habilidades y capacidades, y las menores la situación económica y la seguridad social. No se presentaron diferencias significativas según sexo en la satisfacción personal con los diferentes aspectos de la vida.

Conclusiones El aporte al conocimiento es evidenciar la necesidad de incorporar lo subjetivo, la opinión, la percepción y la valoración que de su propia vida tenga cada persona, como un componente fundamental en la valoración individual de la calidad de vida.

Palabras Clave: Calidad de vida, satisfacción personal, género (fuente: DeCS, BIREME).

\section{ABSTRACT}

Objective Determining personal satisfaction as the main component of the quality of life of men and women aged 20 to 64 in Medellin during 2005. 
REVISTADE SALUD PÚBLICA·Volumen 9 (4), Diciembre 2007

\begin{abstract}
Methods This was a cross/sectional descriptive population study. Its primary source of information consisted of two samples (659 men and 683 women) representing 523705 and 651704 people respectively, according to population expansion factors, selected via probabilistic complex sampling, stratified by socioeconomic level, clustering, city blocks, houses and people.

Results The quality of life for adults in Medellin is mainly represented by personal and economic aspects, their housing's physical environment and social security. The aspects analysed in this study were evaluated above $75 \%$; personal abilities and capability produced the greatest satisfaction, whilst the economic situation and social security produced least satisfaction. There were no significant differences according to gender regarding personal satisfaction concerning various aspects of life.

Conclusions This study's contribution to knowledge lies in making evident the need to integrate each person's subjectivity, opinions and individual perception of his/her own life, since these are the main components in evaluating an individual's quality of life.
\end{abstract}

Key Words: Quality of life, personal satisfaction, gender identity (source: $\mathrm{MeSH}$, NLM).

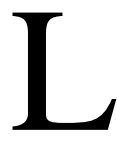
a satisfacción personal es la satisfacción con la vida y está relacionada con la valoración individual de la calidad de vida, regida por apreciaciones subjetivas y objetivas donde se trasciende lo económico y se mira la percepción, opinión, satisfacción y expectativas de las personas (1). Así, para determinar la calidad de vida se requieren referentes de contraste, diversos modos de vida, aspiraciones, ideales e idiosincrasias de los conjuntos sociales, para distinguir eslabones y magnitudes, pudiendo así dimensionar las respectivas variaciones entre unos y otros sectores de la población. La calidad de vida es una construcción histórica y cultural de valores, sujeta a las variables de tiempo, espacio e imaginarios, con los singulares grados y alcances de desarrollo de cada época y sociedad (2).

Actúa en dos ámbitos: individual o privada y colectiva o pública (3). Desde lo individual es importante mirar: percepción, sentido de vida, utilidad, valoración, felicidad, satisfacción de necesidades y demás aspectos subjetivos que son difícilmente cuantificables, pero que hacen que una vida tenga calidad con responsabilidad moral. Desde lo colectivo es fundamental determinar el contexto cultural en el que vive, crece y se desarrolla un individuo pues en él se concentra un capital humano, que responde a significados que él mismo ha tejido con el apoyo de los demás y que corresponden a la trama de sentido de los eventos de la vida cotidiana, le permiten valorarla al contrastarla con criterios 
colectivamente válidos en la sociedad en que vive. La cultura es un determinante transversal de la calidad de vida de la población adulta, porque influye sobre todos los demás determinantes.

Si se considera a priori que ya existe cobertura de ciertas necesidades básicas para su sobrevivencia, el ser humano inmerso dentro de una sociedad, enmarcado en un lugar determinado y una cultura que ha adquirido mediante la socialización se regula e incluso limita las concepciones de su mundo. En este contexto el sujeto evalúa su vida, más o menos consciente de lo que acontece, puesto que aquel proceso se encuentra mediado por una cantidad de factores anexos a los globales: nivel evolutivo, comparación con otros, historia personal, momento actual, expectativas futuras, entre otras; todo esto se conjuga y le permite la evaluación acorde con dicha vivencia. Pero no se puede olvidar las características personales, factor que se si adiciona a los ya mencionados, complejiza aún más el proceso, desde donde se rescata la subjetividad o forma de concebir el mundo.

Lo anteriormente expuesto sugiere que el concepto de calidad de vida hace referencia al mundo ideológico de pensamientos, sensaciones, satisfacciones y subjetividades; tiene sentido para cada persona según la escala valorativa de cada necesidad satisfecha. El modo de comprender la vida humana difiere de una cultura a otra e igual ocurre con la valoración de la calidad de vida; el ser humano interpreta desde un determinado universo de símbolos, representaciones y creencias que hay que enmarcarlos en contextos y tiempos determinados, por lo que desde esta perspectiva no se puede generalizar un único concepto, pues responde a significados culturales, adicionado al sentido que tiene la vida para cada persona y a la valoración teológica que cada persona tenga de ella (4).

La Organización Mundial de la Salud (OMS) definió la calidad de vida como "la percepción individual de la propia posición en la vida dentro del contexto del sistema cultural y de valores en que se vive y en relación con sus objetivos, esperanzas, normas y preocupaciones" (5). Es un concepto coherente con la definición de salud de la misma organización, que incorpora las áreas física, psicológica, nivel de independencia, relaciones sociales, creencias personales y relaciones con el ambiente (6).

Partiendo de estas definiciones, se buscó determinar la satisfacción personal como el principal componente de la calidad de vida de los hombres y las mujeres de 20 a 64 años de la ciudad de Medellín en el año 2005, y hace parte de los 
REVISTADE SALUD PÚBLICA• Volumen 9 (4), Diciembre 2007

resultados de la investigación sobre "Aspectos de la calidad de vida de la población adulta: comparativo por sexo. Medellín 2005" (7). Para ello se entendió la calidad de vida como un fenómeno social complejo y un proceso de percepción personal del nivel de bienestar alcanzado a través de la combinación de las condiciones de vida (variables objetivas que se pueden medir independientemente de los sentimientos) y el grado de satisfacción con la vida (variables subjetivas que dependen de los sentimientos y sólo se pueden mirar a través de los interesados) (8).

\section{MATERIALES Y MÉTODOS}

Se diseñó un estudio poblacional descriptivo de componente transversal, con fuente de información primaria, constituida 659 hombres y 683 mujeres, quienes a través de factores de expansión poblacional representaron a 523705 y 651 704, respectivamente. La población de referencia fueron los 2088245 habitantes de la ciudad de Medellín en el año 2005, según las proyecciones poblacionales del Departamento Administrativo de Planeación Municipal (9); como población de estudio se tomaron los adultos entre 20 y 64 años residentes en la zona urbana: 549214 hombres y 696504 mujeres.

Las muestras fueron obtenidas mediante un muestreo probabilístico complejo o mixto, con diseño estratificado, por conglomerados y polietápico. Probabilístico porque todos los adultos de 20 a 64 años tuvieron una probabilidad de selección, conocida y mayor que cero; estratificado según estrato socioeconómico de la vivienda; por conglomerados construidos por las manzanas, según la distribución urbana de la ciudad; polietápico con selección de manzanas, viviendas de cada manzana y personas, hombre o mujer, en el rango de edad estudiado.

Para el cálculo del tamaño muestral de cada grupo (hombres y mujeres), se utilizó la fórmula para una proporción en población finita con un nivel de confianza $1-\propto=95 \%$, un error del $4 \%$ y con $p=0,5$ pues no se conoce la proporción de población adulta que goza de buena calidad de vida, quedando conformada por 600 personas de cada sexo. Para efectos de controlar las pérdidas en la recolección de la información por no respuesta, viviendas deshabitadas, rechazo a responder la encuesta, conflicto urbano, entre otros, cada muestra se incrementó en un 12,5 \% el cual le confiere más validez a la prueba, quedando constituida por 675 personas cada una.

En el diseño muestral, se utilizó la información del Departamento Administrativo de Planeación Municipal (10) y Secretaría de Hacienda Municipal 
(11) para el año 2002: la ciudad tenía en su zona urbana, 16 comunas, 11510 manzanas, seis estratos socioeconómicos, 592641 viviendas, 51 viviendas por manzana y tres personas por vivienda, en promedio.

El instrumento de recolección de información consistió en un cuestionario (encuesta), con preguntas en escalas de cinco niveles, diseñado por los investigadores, tomando como referencia instrumentos genéricos utilizados en la valoración de la calidad de vida en población general. Fue aplicado por encuestadores contratados por la Universidad de Antioquia, previo el consentimiento informado y los aspectos éticos observados en la Resolución 8430 de 1993 del Ministerio de Salud de Colombia. El análisis fue univariado y bivariado, complementado con intervalos de confianza del $95 \%$ (IC95\%).

En el proceso de estimación se utilizó el factor básico de expansión el cual restituye aproximadamente los valores absolutos del universo estudiado, corrigiendo el efecto de las diferentes probabilidades, ajustadas por composición familiar y sexo que corrigen el eventual sesgo y permite mantener el objetivo de inferir los resultados a la población de estudio.

\section{RESULTADOS}

La satisfacción del ser humano con lo que lo rodea, está determinado por la forma en que se perciba a sí mismo; en este sentido, el 92,8 \% está satisfecho consigo mismo. Con sus relaciones personales la satisfacción es del 91,4 \% (IC95\%: 89,7-93,1), con las relaciones afectivas es el 86, 2\% (IC95\%: 84,388,2 ) y con las relaciones sexuales, el 88,1 \% (IC95\%: 86,1-90,2). En todos los casos los hombres aventajan en satisfacción a las mujeres (Tabla 1).

De los aspectos personales que más proporcionan satisfacción a la vida son las habilidades que se tienen para realizar las actividades de la vida, tanto para los hombres como para las mujeres y las menores son: tiempo dedicado al descanso y, tiempo y calidad del sueño reparador. El 90,6 \% están satisfechos con su habilidad, destreza y pericia para desempeñarse en el campo laboral puesto que hacen parte de la fuerza laboral de la ciudad y consideran que están capacitados para desempeñarse en el sector productivo del país.

En general, la situación económica actual genera satisfacción al 54,6\% (IC95\%: 51,7-57,5) y esta reflejando un alto grado de insatisfacción pues los adultos, no se sienten contentos con sus ingresos, patrimonio, actividad habitual, contratación y sus obligaciones familiares, y no se observa diferencia en la 
REVISTADE SALUD PÚBLICA· Volumen 9 (4), Diciembre 2007

percepción según sexo, pues afecta su propio bienestar, la familia y la sociedad. Los niveles de satisfacción guardan una lógica razonable, al encontrarse que los jubilados están más satisfechos mientras los más insatisfechos son los desempleados e inactivos laboralmente puesto que de ello derivan el cubrimiento de las necesidades de subsistencia.

Tabla 1. Satisfacción personal de la población adulta. Medellín, 2005

\begin{tabular}{lcccc}
\hline \multirow{2}{*}{ Caracteristica } & \multicolumn{2}{c}{ Satisfacción } & \multicolumn{2}{c}{ Insatisfacción } \\
\cline { 2 - 5 } & Hombres & Mujeres & Hombres & Mujeres \\
\hline Habilidades y capacidades & 95,7 & 95,8 & 4,3 & 22,9 \\
Consigo mismo & 93,8 & 92,1 & 6,2 & 21,2 \\
Relaciones personales & 92,8 & 90,2 & 7,2 & 14,6 \\
Relaciones sexuales & 89,6 & 87,0 & 10,4 & 13,0 \\
Relaciones afectivas & 87,3 & 85,4 & 12,7 & 9,8 \\
Estado de salud & 86,8 & 85,3 & 13,2 & 14,7 \\
Tiempo dedicado al sueño & 80,4 & 78,8 & 19,6 & 7,9 \\
Tiempo de descanso & 76,6 & 77,1 & 23,4 & 22,9 \\
Calidad de los servicios de & 77,8 & 77,0 & 22,2 & 23,0 \\
salud & & & & \\
Acceso a los servicios de & 77,2 & 78,0 & 22,8 & 22,0 \\
salud & & & & \\
Atención en los servicios de & 76,5 & 78,0 & 23,5 & 22,0 \\
salud & 76,4 & 23,6 & 75,2 & 24,8 \\
Relaciones sociales & 54,7 & 45,3 & 54,5 & 45,5 \\
\hline Situación económica & & &
\end{tabular}

La satisfacción social está mirada desde las percepciones, preocupaciones y apoyo recibido por la familia como red de apoyo social primario que le brinda el soporte moral al adulto para enfrentar las diferentes situaciones de la vida diaria y con el soporte moral que brinda la red de apoyo secundario constituida por amigos y por último el soporte social que brindan los grupos de organización social, comunitario, de salud o político que brindan acompañamiento al adulto de la ciudad. En cuanto al apoyo recibido de su familia, la satisfacción es del 88,9 \% (IC95\%: 87,2-90,7) y de los amigos el 80,6\%. Con el lugar donde vive, la satisfacción es del 88,7 \% (IC95\%: 86,8-90,7) y no existe mayor diferencia entre hombres y mujeres; con el vecindario, la satisfacción es del 87,3 \% y con el medio de transporte el 91,3 \% (IC95\%: 89,5-93).

Con su seguridad social, la satisfacción con el estado de salud es del $86 \%$ (IC95\%: 84-88). Con la atención recibida en los servicios de salud, la satisfacción es del 77,4\% (IC95\%: 74,9-79,8), con el acceso a los servicios el 77,6 \% (IC95\%: 75,1-80,1) y con la calidad del mismo el 77,3 \% (IC95\%: 74,779,9). Por sexo, se encontró que los hombres están menos satisfechos con la atención recibida que las mujeres (Tabla 1 ). 


\section{DISCUSIÓN}

En esta investigación se abordó la calidad de vida de hombres y mujeres de 20 a 64 años de la ciudad, con el deseo implícito el deseo de ahondar en la igualdad de satisfacciones individuales y personales, según su determinación biológica; situaciones entre el hombre y la mujer basadas en la división, el rol y el poder que ejercen en la sociedad cada uno de ellos. Cuando se habla de igualdad, es ante todo, igualdad de derechos y oportunidades que es necesario establecer por medio de normas, justamente porque los seres humanos son diferentes entre sí. A este respecto la Constitución Nacional dice: "Todas las personas nacen libres e iguales ante a ley, recibirán la misma protección y trato de las autoridades y gozarán de los mismos derechos, libertades y oportunidades sin ninguna discriminación por razones de sexo, raza, origen nacional o familiar, lengua, religión, opinión política o filosófica" (art.13) (12), de aquí se desprende una clara y contundente afirmación sobre el carácter fundamental del derecho a la igualdad, como valor fundante de un Estado social de derecho y de la concepción dignificante del ser humano.

Estos principios constitucionales no hablan directamente del derecho del ser humano a tener una vida con calidad acorde con sus aspiraciones, deseos y necesidades; pero la calidad de las condiciones y satisfacciones están íntimamente ligados a la vida mediados por un acto de razón, no solo según la escala de valores, aspiraciones y expectativas personales ni de su universo de símbolos, representaciones y creencias, enmarcados en contextos y tiempos determinados, sino que cada persona evalúa su vida según su propia subjetividad y su propia escala de valores.

Por lo tanto, la sola existencia del hombre le atribuye el derecho a exigir y a obtener la vigencia de todas las garantías necesarias para asegurar una vida digna: una existencia adecuada, proporcional y racional al reconocimiento de su esencia como ser pensante; al mismo tiempo, le impone al Estado el deber de adoptar las medidas de protección indispensables para salvaguardar los bienes jurídicos que definen al hombre como persona, es decir, la vida, la dignidad, la integridad, la libertad, la autonomía, etc.

Aquí se explicita la calidad de vida desde un contexto ético, puesto que es el ser humano el que designa una determinada clase de objetos necesarios para su vida, lo individualiza y lo diferencia de los demás, como un acto humano racional y por ende, la calidad de vida es individual y se evalúa desde la subjetividad. También apoya la definición de calidad de vida de la Organización 
REVISTADE SALUD PÚBLICA· Volumen 9 (4), Diciembre 2007

Mundial de la Salud que la considera como una percepción individual según las oportunidades, deseos o preferencias; donde las satisfacciones dadas por los bienes materiales son medios -no fines- del bienestar de las personas (5).

En general, la valoración de la calidad de vida fue satisfactoria y por encima del 75 \%, con excepción de la situación económica actual que alcanzó un 55 \% y la seguridad social con un 64 \%. La satisfacción personal no presenta diferencias estadísticamente significativas según sexo; en casi todo los aspectos, la satisfacción es superior en la población masculina mientras la femenina valoró con mayor satisfacción la situación económica y la seguridad social.

El aporte al conocimiento sobre la valoración de la calidad de vida, es mostrar la necesidad de incorporar lo subjetivo, la opinión, la percepción individual y la valoración que de su propia vida tenga cada persona. Incluir los aspectos importantes para la vida humana y de satisfacción personal, como las relaciones interpersonales que lo hacen un ser social, la responsabilidad moral del comportamiento individual; la satisfacción de las diversas necesidades humanas, la valoración de cada necesidad y su correspondiente satisfactor; los derechos fundamentales inherentes al ser como libertad, igualdad y disfrute de las condiciones de vida que le permitan llevar una vida digna; dignidad que se busca a través del bienestar

\section{REFERENCIAS}

1. European Fundation for the improvement of living and working conditions. Quality of life in Europe. Alemania: Publications Office; 2005.

2. Espinosa O. Apuntes sobre calidad de vida, desarrollo sostenible y sociedad de consumo: una mirada desde América Latina. Rev Contribuciones de la Fundación Konrad Adenauer deAlemaniay el CIEDLA1999;63(3):119-48.

3. Rodado NC, Grijalba de RE. La tierra cambia de piel: Una visión integral de la calidad de vida. 2a. ed. Bogotá, D.C: Editorial Planeta Colombiana, S.A; 2001.

4. Lugones BM. Algunas consideraciones sobre la calidad de vida. Rev Cubana Med Gen Integr 2002;18(4): 287-289.

5. Organización Mundial de la Salud, Programa Envejecimiento y Ciclo Vital. Envejecimiento activo: un marco político. Rev Esp Geriatr Gerontol 2002;37(S2):74-105.

6. Boladeras CM. Calidad de vida y principios bioéticos. En: Colección Bíos y Ethos, editor. Bioética y Calidad de Vida. Bogotá, D.C: Ediciones El Bosque; 2000. p. 21-54.

7. Cardona AD, Agudelo GH. La flor de la vida: pensemos en el adulto. Aspectos de la calidad de vida de la población adulta. Medellín, 2005. Medellín: Facultad Nacional de Salud Pública, Universidad de Antioquia; 2006. 
8. Cardona AD, Estrada RA, Agudelo GH. Envejecer nos Toca a todos. Medellín: Facultad Nacional de Salud Pública, Universidad de Antioquia; 2003.

9. Departamento Administrativo de Planeación Municipal, Unidad de Clasificación Socioeconómica y Estratificación. Proyección de Población. Medellín: Alcaldía; 2005.

10. Departamento Administrativo de Planeación Municipal, Unidad de Clasificación Socioeconómica y Estratificación. Estimación del número de instalaciones de energía en viviendas del Municipio de Medellín, por estrato socioeconómico, según barrio, comuna y corregimiento de localización. Medellín: Alcaldía; 2002.

11. Secretaría de Hacienda Municipal, Oficina de Catastro. Dirección de ubicación de las manzanas de la ciudad de Medellín. Medellín: Alcaldía; 2005.

12. Congreso de la República de Colombia. Constitución Política de Colombia. Bogotá: El Congreso; 1991. 